\title{
GENERALIZED CONDITIONAL EXPECTATIONS
}

\author{
WILLIAM E. HORNOR
}

(Communicated by Andrew M. Bruckner)

\begin{abstract}
In this paper, we state conditions sufficient for the existence of conditional expectations. Given a measure space $(X, \Sigma, \mu)$ and a $\sigma$-subalgebra $\mathscr{A} \subset \Sigma$, we give conditions on $\mathscr{A}$ which insure that for every real-valued $\Sigma$ measurable function $f$ there exists a $\mathscr{A}$-measurable function $E(f)$ such that $\int g f d \mu=\int g E(f) d \mu$ for every $\mathscr{A}$-measurable function $g$ for which the left integral exists. These conditions entail a notion of "fineness" of the subalgebra $\mathscr{A}$ and a "completeness" property of $(X, \mathscr{A}, \mu)$. We then introduce a notion of generalized conditional expectation which requires only the former condition.
\end{abstract}

\section{INTRODUCTION}

In this paper we present a natural extension of the concept of conditional expectation in which the integrability properties of the functions to be conditioned play no role. Our work differs markedly from previous extensions, such as that of Brunk [2], in which the main theme was a broadening of the class of measure spaces under consideration. We do concern ourselves with maintaining a high degree of generality, and the restrictions placed on the measure spaces admitted are rather minimal.

Given a measure space $(X, \Sigma, \mu)$ and a $\sigma$-subalgebra $\mathscr{A} \subset \Sigma$, we seek conditions on $\mathscr{A}$ under which every $\Sigma$-measurable function $f: X \rightarrow \mathbf{R}$ is $\mathscr{A}$ conditionable in the sense that there exists a $\mathscr{A}$-measurable function $E(f)$ such that

$$
\int g f d \mu=\int g E(f) d \mu
$$

for every $\mathscr{A}$-measurable function $g$ for which the integral on the left exists. Any such function $E(f)$ is called a conditional expectation of $f$ relative to the $\sigma$-subalgebra $\mathscr{A}$. We establish sufficient conditions for the existence of conditional expectations involving the concepts of localizability and totality of the $\sigma$-subalgebra $\mathscr{A}$ in $\Sigma$. In Section 4, we drop our localizability assumption

Received by the editors September 15, 1993 and, in revised form, January 19, 1994 and May 4, 1994; this paper was presented at the Joint AMS-CMS Meeting in Vancouver, British Columbia, in August 1993.

1991 Mathematics Subject Classification. Primary 28A99. 
and demonstrate that some notion of conditional expectation exists provided only that the $\sigma$-subalgebra $\mathscr{A}$ is total in $\Sigma$.

\section{Definitions AND NOTATION}

Throughout this paper $(X, \Sigma, \mu)$ will denote a complete measure space and $\mathscr{A}$ a $\sigma$-subalgebra such that the restriction $\mu_{\mathscr{A}}$ of $\mu$ to $\mathscr{A}$ is complete. The collection of real-valued $\mathscr{A}$-measurable functions will be written as $L^{0}(\mathscr{A})$, where functions which are equal a.e.- $\mu$ are regarded as equal. We write $L_{+}^{0}(\mathscr{A})$ for the subcollection of $L^{0}(\mathscr{A})$ consisting of nonnegative functions. For each $f \in L_{+}^{0}(\Sigma)$, we define a measure $\nu=\nu_{f}$ on $\mathscr{A}$ by

$$
\nu(A)=\int_{A} f d \mu
$$

for every $A \in \mathscr{A}$. The collection of all $\mathscr{A}$-measurable sets of finite $\mu$-measure is denoted $\mathscr{A}_{0}$.

Definition 1. A $\sigma$-subalgebra $\mathscr{A}$ of $\Sigma$ is said to be total in $\Sigma$ if for each $B \in \Sigma$

$$
\mu(B)=\sup \left\{\mu\left(B \cap A_{0}\right): A_{0} \in \mathscr{A}_{0}\right\} .
$$

Definition 2. If $\lambda$ is a measure on $\mathscr{A}$, we say that $\lambda$ is accessible with respect to $\mu$ if for each $A \in \mathscr{A}$

$$
\lambda(A)=\sup \left\{\lambda\left(A_{0}\right): A_{0} \subset A, A_{0} \in \mathscr{A}_{0}\right\} .
$$

For each $\Sigma$-measurable function $f$, the support of $f$ is defined as $\{x \in$ $X: f(x) \neq 0\}$ and is denoted by $\operatorname{supp}(f)$. The extended reals will be denoted by $\overline{\mathbf{R}}$.

\section{LOCALIZABILITY AND CONDITIONAL EXPECTATION}

Lemma 3, whose proof of straightforward, expresses the relationship between the totality of $\mathscr{A}$ in $\Sigma$ and the accessibility of the measures $\nu_{f}$ with respect to $\mu_{\mathscr{A}}$.

Lemma 3. The $\sigma$-subalgebra $\mathscr{A}$ is total in $\Sigma$ if and only if for each $f \in L_{+}^{0}(\Sigma)$ the corresponding measure $\nu_{f}$ on $\mathscr{A}$ is accessible with respect to $\mu_{\mathscr{A}}$.

Lemma 4. Suppose $\mathscr{A}$ is total in $\Sigma$ and $\left(X, \mathscr{A}, \mu_{\mathscr{A}}\right)$ is localizable. Then for any $f \in L_{+}^{0}(\Sigma)$ there exists a nonnegative, (extended) real-valued, $\mathscr{A}$ measurable function $\mathscr{E}(f)$ such that $\left(X, \mathscr{A},(f d \mu)_{\mathscr{A}}\right)=\left(X, \mathscr{A}, \mathscr{E}(f) d \mu_{\mathscr{A}}\right)$. Proof. By Lemma 3, the measure $\nu_{f}$ corresponding to $f$ is accessible with respect to $\mu_{\mathscr{A}}$, and the function $\mathscr{E}(f)$ is the Radon-Nikodym derivative $\frac{d \nu_{f}}{d \mu_{\mathscr{A}}}$. See [1].

If $\mathscr{A}$ and $f$ satisfy the hypotheses of Lemma 4 , set $\mathscr{E}_{0}(f)=\chi_{S} \mathscr{E}(f)$ where $S=S_{f}=\{\mathscr{E}(f)<\infty\}$. We remark that the measure $A \mapsto \int_{A} \mathscr{E}_{0}(f) d \mu$ is the semi-finite part $\nu_{0}$ of $\nu_{f}$.

Theorem 5. Suppose $\mathscr{A}$ is a complete $\sigma$-subalgebra of $\Sigma$ suck that $\mathscr{A}$ is total in $\Sigma$ and $\left(X, \mathscr{A}, \mu_{\mathscr{A}}\right)$ is localizable. Then there exists a conditional expectation operator $E: L^{0}(\Sigma) \rightarrow L^{0}\left(X, \mathscr{A}, \mu_{\mathscr{A}}, \overline{\mathbf{R}}\right)$ such that for each $f \in L^{0}(\Sigma)$ we have

$$
\int g f d \mu=\int g E(f) d \mu_{\mathscr{A}}
$$


for every $g \in L^{1}\left(X, \mathscr{A},(|f| d \mu)_{\mathscr{A}}\right)$. The operator $E$ is faithful in the sense that if $f \in L_{+}^{0}(\Sigma)$ and $E(f) \equiv 0$, then $f \equiv 0$.

Proof. Suppose $\mathscr{A}$ and $f$ satisfy the hypotheses of the theorem, and let $g \in$ $L^{1}\left(X, \mathscr{A},(|f| d \mu)_{\mathscr{A}}\right)$. Then

$$
\int g|f| d \mu=\int g \mathscr{E}(|f|) d \mu=\int g \mathscr{E}_{0}(|f|) d \mu_{\mathscr{A}} .
$$

Consider the space $\left(X, \mathscr{A}, \nu_{0}\right)=\left(X, \mathscr{A}, \mathscr{E}_{0}(|f|) d \mu_{\mathscr{A}}\right)$. Since $\mathscr{E}_{0}(|f|)$ is finitevalued and $\left(X, \mathscr{A}, \mu_{\mathscr{A}}\right)$ is semi-finite and localizable, $\left(X, \mathscr{A}, \nu_{0}\right)$ has these properties as well. Define a linear functional $\Gamma_{f}$ on $L^{1}\left(X, \mathscr{A}, \nu_{0}\right)$ by

$$
\Gamma_{f}(g)=\int_{S_{|,|}} g f d \mu
$$

Note that

$$
\left|\Gamma_{f}(g)\right| \leq \int_{S_{|, f|}}|g||f| d \mu=\int|g| \mathscr{E}_{0}(|f|) d \mu_{\mathscr{A}}=\|g\|_{\nu_{0}}
$$

By the Riesz Representation Theorem, there exists a $\nu_{0}$-unique

$$
\varphi_{f} \in L^{\infty}\left(X, \mathscr{A}, \nu_{0}\right) \text {, }
$$

of norm less than or equal to one and vanishing on $X \backslash \operatorname{supp}\left(\mathscr{E}_{0}(|f|)\right)$, such that

$$
\Gamma_{f}(g)=\int g \varphi_{f} \mathscr{E}_{0}(|f|) d \mu_{\mathscr{A}}
$$

Set $E_{1}(f)=\varphi_{f}^{\mathscr{E}} \mathscr{E}_{0}(|f|), E_{2}(f)=\chi_{s_{|,|}^{c}} \mathscr{E}(|f|)=\infty \cdot \chi_{s_{|,|}}$, and $E(f)=E_{1}(f)+$ $E_{2}(f)=\left(\varphi_{f}+\chi_{S_{|, f|}}\right) \mathscr{E}(|f|)$ for every $f \in L^{0}(\Sigma)$.

If $f \in L_{+}^{0}(\Sigma)$ with $E(f) \equiv 0$, then $S_{|f|}=X$, and $\mathscr{E}(f)=\mathscr{E}_{0}(f)=E(f) \equiv$ 0 . For any $A_{0} \in \mathscr{A}_{0}$ we have $\int \chi_{A} f d \mu=\int \chi_{A} \mathscr{E}(f) d \mu=0$. Since $f \in L_{+}^{0}(\Sigma)$ and $\mathscr{A}$ is total in $\Sigma$, it follows that $f \equiv 0$.

The following example demonstrates that the definition of conditional expectation given (implicitly) in Theorem 5 differs from the classical notion as presented in [4].

Example 6. Consider the probability space $\left(\mathbf{R}^{2}, \Sigma_{2}, \mu\right)$ where $\Sigma_{k}$ denotes the Lebesgue measurable subsets of $\mathbf{R}^{k}$ and $\mu$ is defined by

$$
\mu(A)=\int_{A} \frac{|y|}{4} e^{-|y|(1+|x|)}
$$

for every $A \in \Sigma_{2}$. Set $\mathscr{A}=\left\{\mathbf{R} \times B: B \in \Sigma_{1}\right\}$ and define $f: \mathbf{R}^{2} \rightarrow \mathbf{R}$ by $f(x, y)=x$. According to [4], $f$ is not conditionable in the classical sense. However, using our definition, $f$ is conditionable with $E(f) \equiv 0$. In our notation, we have

$$
\nu_{|f|}(A)=\int_{A} \frac{|x y|}{4} e^{-|y|(1+|x|)} \text { and } g \in L^{1}\left(\mathbf{R}^{2}, \mathscr{A}, \nu_{|f|}\right)
$$

if and only if $g(x, y)=g(y)$ and $g \in L^{1}\left(\mathbf{R}, \Sigma_{1}, \frac{e^{-|y|}}{|y|} d y\right)$. But $\Gamma_{f}(g) \equiv 0$ for all such $g$, and hence $\varphi_{f} \equiv 0$. Thus $f$ is, in a sense, orthogonal to $\mathscr{A}$. 


\section{GeNERALIZED CONDITIONAL EXPECTATION}

In this final section, we drop our assumption that $\left(X, \mathscr{A}, \mu_{A}\right)$ is localizable while retaining our assumption that $\mathscr{A}$ is total in $\Sigma$. Under these conditions, for each $\Sigma$-measurable $f$, there exists a net of $\mathscr{A}$-measurable functions which serves as a generalized conditional expectation is a sense made precise below.

Let $\mathscr{A}_{\sigma}=\left\{A \in \mathscr{A}: A\right.$ is of $\sigma$-finite $\mu_{\mathscr{A}}$-measure $\}$. Throughout this section, $f$ will represent a fixed but arbitrary real-valued $\Sigma$-measurable function. As above, we define a measure $\nu=\nu_{|f|}$ on $\mathscr{A}$ by $\nu(A)=\int_{A}|f| d \mu$. For each $A_{\sigma} \in$ $\mathscr{A}_{\sigma}$ we define measures $\mu_{A_{\sigma}}$ and $\nu_{A_{\sigma}}$ on $\mathscr{A}$ by setting $\mu_{A_{\sigma}}(A)=\mu\left(A \cap A_{\sigma}\right)$ and $\nu_{A_{\sigma}}(A)=\nu\left(A \cap A_{\sigma}\right)$ for every $A \in \mathscr{A}$. By the Radon-Nikodym Theorem, for each $A_{\sigma} \in \mathscr{A}_{\sigma}$ there exists a nonnegative, extended real-valued, $\mathscr{A}$-measurable function $\mathscr{E}\left(|f|, A_{\sigma}\right)$, vanishing off $A_{\sigma}$ and uniquely determined on $A_{\sigma}$, such that

$$
\nu_{A_{\sigma}}(A)=\int_{A} \mathscr{E}\left(|f|, A_{\sigma}\right) d \mu
$$

for every $A \in \mathscr{A}$. Equivalently, $\left(X, \mathscr{A}, \nu_{A_{\sigma}}\right)=\left(X, \mathscr{A}, \mathscr{E}\left(|f|, A_{\sigma}\right) d \mu\right)$. Now setting $S^{A_{\sigma}}=\left\{\mathscr{E}\left(|f|, A_{\sigma}\right)<\infty\right\}$ and $\mathscr{E}_{0}\left(|f|, A_{\sigma}\right)=\chi_{S^{A_{\sigma}}} \mathscr{E}\left(|f|, A_{\sigma}\right)$, we have

$$
L^{1}\left(\nu_{A_{\sigma}}\right)=L^{1}\left(\mathscr{E}_{0}\left(|f|, A_{\sigma}\right) d \mu\right) .
$$

For each $A_{\sigma} \in \mathscr{A}_{\sigma}$, define a measure $\nu_{A_{\sigma}}^{0}$ on $\mathscr{A}$ by $\nu_{A_{\sigma}}^{0}(A)=\int_{A} \mathscr{E}_{0}\left(|f|, A_{\sigma}\right) d \mu$. If $A_{\sigma}, A_{\sigma}^{\prime} \in \mathscr{A}_{\sigma}$, then for any $\mathscr{A}$-measurable $A \subset A_{\sigma} \cap A_{\sigma}^{\prime}$ we have

$$
\int_{A} \mathscr{E}_{0}\left(|f|, A_{\sigma}\right) d \mu=\nu_{A_{\sigma}}^{0}(A)=\nu_{A_{\sigma}^{\prime}}^{0}(A)=\int_{A} \mathscr{E}_{0}\left(|f|, A_{\sigma}^{\prime}\right) d \mu,
$$

and it follows that $\mathscr{E}_{0}\left(|f|, A_{\sigma}\right)$ and $\mathscr{E}_{0}\left(|f|, A_{\sigma}^{\prime}\right)$ agree on $A_{\sigma} \cap A_{\sigma}^{\prime}$. So for any countable subcollection $\left\{A_{\sigma}^{n}\right\}$ of $\mathscr{A}_{\sigma}$, we have

$$
\chi_{A_{\sigma}^{n} \mathscr{E}_{0}}\left(|f|, \bigcup A_{\sigma}^{k}\right)=\mathscr{E}_{0}\left(|f|, A_{\sigma}^{n}\right) .
$$

Next, for each $A_{\sigma} \in \mathscr{A}_{\sigma}$ define $\Gamma^{A_{\sigma}}$ on $L^{1}\left(\nu_{A_{\sigma}}^{0}\right)$ by

$$
\Gamma^{A_{\sigma}}(g)=\int_{S^{A_{\sigma}}} g f d \mu
$$

Now

$$
\left|\Gamma^{A_{\sigma}}(g)\right| \leq \int_{S^{A_{\sigma}}}|g||f| d \mu=\int|g| d \nu_{A_{\sigma}}^{0}=\|g\|_{\nu_{A_{\sigma}}^{0}},
$$

so by the Riesz Representation Theorem there exists a $\varphi\left(f, A_{\sigma}\right) \in L^{\infty}\left(\nu_{A_{\sigma}}^{0}\right)$, of norm at most one, vanishing off and uniquely determined on $A_{\sigma}$, such that

$$
\Gamma^{A_{\sigma}}(g)=\int g \varphi\left(f, A_{\sigma}\right) d \nu_{A_{\sigma}}^{0}=\int g \varphi\left(f, A_{\sigma}\right) \mathscr{E}_{0}\left(|f|, A_{\sigma}\right) d \mu .
$$

If $A_{\sigma}, A_{\sigma}^{\prime} \in \mathscr{A}_{\sigma}$ and $A \in \mathscr{A}$ with $A \subset A_{\sigma} \cap A_{\sigma}^{\prime}$, then for any $g \in L^{1}\left(\nu_{A}^{0}\right)$ with $g \equiv 0$ off $A$, we have

$\int g \varphi\left(f, A_{\sigma}\right) \mathscr{E}_{0}\left(|f|, A_{\sigma}\right) d \mu=\Gamma^{A_{\sigma}}(g)=\Gamma^{A_{\sigma}^{\prime}}(g)=\int g \varphi\left(f, A_{\sigma}^{\prime}\right) \mathscr{E}_{0}\left(|f|, A_{\sigma}^{\prime}\right) d \mu$,

and we see that $\varphi\left(f, A_{\sigma}\right)=\varphi\left(f, A_{\sigma}^{\prime}\right)$ on $A_{\sigma} \cap A_{\sigma}^{\prime}$. Set $E_{A_{\sigma}}(f)=\varphi\left(f, A_{\sigma}\right) \times$ $\mathscr{E}_{0}\left(|f|, A_{\sigma}\right)$ for every $A_{\sigma} \in \mathscr{A}_{\sigma}$. In the terminology of [3], $\left\{E_{A_{\sigma}}(f): A_{\sigma} \in \mathscr{A}_{\sigma}\right\}$ is a measurable quasifunction relative to the family $\left\{\mu_{A_{\sigma}}: A_{\sigma} \in \mathscr{A}_{\sigma}\right\}$ of measures 
on $(X, \mathscr{A})$. If $\left\{A_{\sigma}^{n}\right\}$ is any countable subcollection of $\mathscr{A}_{\sigma}$ and $A_{\sigma}=\bigcup A_{\sigma}^{n}$, we have

$$
\chi_{A_{\sigma}^{n}} E_{A_{\sigma}}(f)=E_{A_{\sigma}^{n}}(f)
$$

If $g \in L^{1}(\nu)$, then $\operatorname{supp}(g f)$ is a $\sigma$-finite set and since $\mathscr{A}$ is total in $\Sigma$, we can choose a sequence $\left\{A_{0}^{n}\right\}$ of elements of $\mathscr{A}_{0}$ such that $\left\{\chi_{A_{0}^{n}} g f\right\}$ converges to $g f$ in measure. Hence

$$
\int g f d \mu=\lim _{n} \int \chi_{A_{0}^{n}} g f d \mu=\lim _{n} \int g E_{A_{0}^{n}}(f) d \mu=\int g E_{A_{\sigma}}(f) d \mu
$$

where $A_{\sigma}=\bigcup A_{0}^{n}$.

The preceding discussion is summarized in the following theorem.

Theorem 7. Suppose $(X, \Sigma, \mu)$ is a measure space and $\mathscr{A}$ is a total $\sigma$-subalgebra of $\Sigma$. Then for each real-valued $\Sigma$-measurable function $f$ there exists $a$ $\mathscr{A}_{\sigma}$-net $\left\{E_{A_{\sigma}}(f)\right\}$ of real-valued $\mathscr{A}$-measurable functions such that

$$
\int g f d \mu=\lim _{A_{\sigma}} \int g E_{A_{\sigma}}(f) d \mu
$$

for every $\mathscr{A}$-measurable $g$ for which the left integral exists.

The net $\left\{E_{A_{\sigma}}(f)\right\}$ is a generalized conditional expectation for $f$ relative to $\mathscr{A}$. We mention in closing that the family $\mathscr{A}_{\sigma}$ can be replaced with $\mathscr{A}_{0}$, or, if we make reference to Theorem 5 , the collection $\mathscr{A}_{\mathscr{L}}=\left\{A \in \mathscr{A}:\left(X, \mathscr{A}, \mu_{A}\right)\right.$ is localizable $\}$. In the first case, $\left\{E_{A_{0}}(f)\right\}$ is a cross-section as defined in [5] or [6].

\section{REFERENCES}

1. A. Brown and C. Pearcy, Introduction to operator theory I, Springer-Verlag, New York, 1977.

2. H. B. Brunk, On an extension of the concept conditional expectation, Proc. Amer. Math. Soc. 14 (1963), 298-304.

3. E. J. McShane, Families of measures and representations of algebras of operators, Trans. Amer. Math. Soc. 102 (1962), 328-345.

4. M. M. Rao, Measure theory and integration, Wiley, New York, 1987.

5. A. C. Zaanen, Integration, North-Holland, Amsterdam, 1967.

6. _ The Radon-Nikodym theorem, I-II, Indag. Math. 23 (1961), 157-187.

Department of Mathematics, University of Southern Mississippi, Hattiesburg, MissisSIPPI 39406 